\title{
- OPEN ACCESS \\ Antibiotic therapy for pelvic inflammatory disease: an abridged version of a Cochrane systematic review and meta-analysis of randomised controlled trials
}

\author{
Ricardo F Savaris, ${ }^{1}$ Daniele G Fuhrich, ${ }^{1}$ Rui V Duarte, ${ }^{2}$ Sebastian Franik, ${ }^{3}$ \\ Jonathan D C Ross ${ }^{4}$
}

\begin{abstract}
- Additional material is published online only. To view please visit the journal online (http://dx.doi.org/10.1136/ sextrans-2018-053693)

${ }^{1}$ Ginecologia e Obstetricia, Universidade Federal do Rio Grande do Sul-FAMED, Porto Alegre, Brazil

${ }^{2}$ Liverpool Reviews and Implementation Group, University of Liverpool, Liverpool, UK

${ }^{3}$ Department of Gynaecology and Obstetrics, Münster University Hospital, Münster, Germany

${ }^{4}$ Whittall Street Clinic, University Hospital Birmingham NHS Foundation Trust, Birmingham,
\end{abstract}

\section{Correspondence to} Rui V Duarte, Liverpool Reviews and Implementation Group, University of Liverpool, Liverpoo L69 3GB, UK; rui.duarte@ liverpool.ac.uk

Received 21 May 2018 Revised 29 August 2018 Accepted 5 September 2018 Published Online First 19 October 2018

\section{SLinked}

- http://dx.doi.org/10.1136/ sextrans-2018-053762

Check for updates

(C) Author(s) (or their employer(s)) 2019. Re-use permitted under CC BY. Published by BMJ.

To cite: Savaris RF

Fuhrich DG, Duarte RV

et al. Sex Transm Infect

2019:95:21-27.

\section{ABSTRACT}

Objective To assess the effectiveness and safety of antibiotic regimens used to treat pelvic inflammatory disease (PID).

Design This is a systematic review and meta-analysis of randomised controlled trials (RCTs). Risk of bias was assessed using the criteria outlined in the Cochrane guidelines. Quality of evidence was assessed using the Grading of Recommendations Assessment, Development and Evaluation.

Data sources Eight electronic databases were searched from date of inception up to July 2016. Database searches were complemented by screening of reference lists of relevant studies, trial registers, conference proceeding abstracts and grey literature. Eligibility criteria RCTs comparing the use of antibiotics with placebo or other antibiotics for the treatment of PID in women of reproductive age, either as inpatient or outpatient treatment.

Results We included 37 RCTs (6348 women). The quality of evidence ranged from very low to high, the main limitations being serious risk of bias (due to poor reporting of study methods and lack of blinding), serious inconsistency and serious imprecision. There was no clear evidence of a difference in the rates of cure for mild-moderate or for severe PID for the comparisons of azithromycin versus doxycycline, quinolone versus cephalosporin, nitroimidazole versus no use of nitroimidazole, clindamycin plus aminoglycoside versus quinolone, or clindamycin plus aminoglycoside versus cephalosporin. No clear evidence of a difference between regimens in antibiotic-related adverse events leading to discontinuation of therapy was observed.

Conclusions We found no conclusive evidence that one regimen of antibiotics was safer or more effective than any other for the treatment of PID, and there was no clear evidence for the use of nitroimidazoles (metronidazole) compared with the use of other drugs with activity against anaerobes. More evidence is needed to assess treatments for women with PID, particularly comparing regimens with or without the addition of nitroimidazoles and the efficacy of azithromycin compared with doxycycline.

\section{INTRODUCTION}

Pelvic inflammatory disease (PID) in women is described as an inflammation of the upper genital tract and surrounding structures as a result of ascending infection from the lower genital tractbacteria spread directly from the cervix to the endometrium and on to the upper genital tract. ${ }^{1}$ The signs and symptoms of PID are not specific and may range from asymptomatic to abdominal pain/tenderness, fever, vomiting, dyspareunia and menorrhagia. $^{2}$

The incidence of PID in the UK has been estimated to range between $1.1 \%$ and $1.7 \%$ among women between 16 and 46 years old. ${ }^{3} 4$ Among women with PID, 10\%-20\% may become infertile, 40\% will develop chronic pelvic pain, and $10 \%$ of those who conceive will have an ectopic pregnancy. ${ }^{5-8}$ The cost of pelvic infection has been estimated to exceed US $\$ 2.4$ billion in the USA, and the mean total cost per episode managed as an outpatient is around US\$700. ${ }^{9}$ In the UK, the mean cost of an uncomplicated episode of PID is $£ 163$, considering an average of two outpatient appointments per woman across all settings. ${ }^{10}$

PID requires effective treatment to reduce the incidence of chronic pelvic pain, infertility and ectopic pregnancy. The main intervention for treating acute PID is the use of broad-spectrum antibiotics which cover Chlamydia trachomatis, Neisseria gonorrhoeae and anaerobic bacteria, but the optimal treatment strategy is unclear. A variety of antibiotic regimens and routes of administration (intravenous, intramuscular or oral) have been used, with marked geographical variation. Current practice generally involves the use of multiple agents to provide broad antimicrobial cover, but the best combination of agents is unknown. Guidelines have been produced in the USA, ${ }^{2}$ and in Europe ${ }^{11}$ to guide therapy, but these have not been based on a systematic review. The authors of the current Centers for Disease Control and Prevention (CDC) and International Union against Sexually Transmitted Infections (IUSTI) guidelines state that there is limited evidence for the need to eradicate anaerobes or for the use of alternative regimens, such as azithromycin. ${ }^{211}$

The current review is an abridged version of a Cochrane systematic review and presents the main findings from the primary outcomes and an enhanced discussion section. ${ }^{12}$ This review addresses clinical questions raised in the current guidelines on the treatment of PID $^{211}$ regarding the effectiveness and safety of nitroimidazole, the relative benefits of azithromycin versus doxycycline, the use of quinolones, and the relative benefits of cephalosporins compared with clindamycin plus aminoglycoside, to inform future guideline development and clinical practice. 


\section{METHODS}

We used Cochrane methodology, ${ }^{13}$ following our published protocol. $^{14}$

\section{Methods for identification of studies}

We searched the Cochrane Sexually Transmitted Infections Review Group's Specialised Register, the Cochrane Central Register of Controlled Trials, MEDLINE, MEDLINE In-Process \& Other Non-Indexed Citations, MEDLINE Daily Update, Embase, LILACS and Web of Science up to July 2016. The complete search strategy is available in the Cochrane review. ${ }^{12}$ We screened the reference lists of all identified randomised controlled trials (RCTs) and previous systematic reviews on similar topics for additional relevant articles. Furthermore, we searched trial registers, conference proceeding abstracts and grey literature. We contacted the authors of all RCTs identified by other methods, as well as pharmaceutical companies producing 'antibiotic therapy' for 'pelvic inflammatory disease (PID)'.

\section{Types of studies}

We included RCTs, including those which did not describe their method of randomisation (ie, where the authors stated that treatment was randomised without providing further details). Trials were included irrespective of publication status, publication year or language. We excluded quasi-randomised trials because they produce effects estimates indicating more extreme benefits when compared with RCTs. ${ }^{13}$ We also excluded cross-over and cluster trials.

\section{Selection of studies}

Two review authors (DGF and RVD) performed an initial screen of titles and abstracts retrieved by the search, and we retrieved the full text of all potentially eligible studies. Two review authors (DGF and RVD) independently examined these studies for compliance with the inclusion criteria and selected studies that met these criteria. Disagreements regarding eligibility were resolved by discussion or by consulting a third review author (JR).

\section{Inclusion criteria}

RCTs were included in the review if the women participating in the trial were of reproductive age (14 years of age or older) diagnosed as having acute PID (symptoms for less than 6 weeks) based on clinical findings, laparoscopy, endometrial biopsy, or detectable N. gonorrhoeae or C. trachomatis in the upper genital tract. We limited our review to comparison of drugs in current use that are recommended for consideration by the 2015 CDC guidelines for treatment of PID. ${ }^{2}$

\section{Outcomes}

The primary outcomes were clinical cure according to the criteria defined by the treating physician (eg, resolution or improvement of signs and symptoms related to PID) and antibiotic-related adverse events leading to discontinuation of therapy. For secondary outcomes, please see the full Cochrane systematic review. $^{12}$

Where studies included women with various types of pelvic infection, we considered only women with endometritis, salpingitis, parametritis or oophoritis (not related to labour, delivery, cancer or surgery). Where studies reported multiple time points, we included outcomes at between 14 and 28 days after initiation of treatment.

\section{Data extraction}

Data from each study were extracted independently by two of the three review authors (SF, DGF, RVD) using a data extraction form that the review authors designed and pilot-tested. Disagreements were resolved by consensus or by consulting a fourth review author (JR or RFS). If a study had more than two intervention arms, we included or combined only those that met the predefined inclusion criteria. Where studies had multiple publications, we used the main trial report as the reference and derived additional details from secondary papers. We corresponded with study investigators for further data as required.

\section{Quality of evidence}

For each included trial, three review authors (SF, DGF, RVD) independently assessed the risk of bias using the criteria outlined in the Cochrane guidelines. ${ }^{13}$ Disagreements were resolved by discussion or by involving a third review author (JR or RFS).

Two reviewers (SF, RFS) independently assessed and graded the evidence according to the Grading of Recommendations Assessment, Development and Evaluation (GRADE). ${ }^{15}$ Disagreements were resolved by discussion or by involving a third review author (JR). The GRADE tables are available in the full Cochrane review. $^{12}$

\section{Data synthesis and analysis}

Data analyses were performed using Review Manager V.5.3. ${ }^{16}$ A fixed-effect meta-analysis was used for combining data where it was reasonable to assume that trials were estimating the same underlying treatment effect (ie, where trials were examining the same intervention, and the trials' populations and methods were sufficiently similar). We conducted separate analyses for mild-moderate and severe PID based on the CDC criteria. ${ }^{2}$ If there was clinical heterogeneity sufficient to expect that the underlying treatment effects differed between trials, or if substantial statistical heterogeneity was detected $\left(\mathrm{I}^{2}=40 \%\right.$ or greater), a random-effects meta-analysis was planned to produce an overall summary if a mean treatment effect across trials was considered to be clinically meaningful. For random-effects analyses, the results are presented as the mean treatment effect with $95 \%$ CIs, and the estimates of the $\operatorname{tau}^{2}$ and $\mathrm{I}^{2}$ statistics.

For dichotomous data, the number of events in the control and intervention groups was used to calculate the MantelHaenszel risk ratios (RR). For the number needed to treat for an additional beneficial (NNTB) or harmful (NNTH) outcome, the recommendations given by Altman were followed. ${ }^{17}$ When we observed a treatment effect, we reported the NNTH or NNTB with 95\% CIs. NNTB and NNTH are presented in the GRADE tables in the full Cochrane review. ${ }^{12}$ When possible, we performed analysis based on intention to treat (ITT). When information for an ITT analysis was not available, we used the results provided by the authors.

We undertook the following sensitivity analysis to investigate whether our conclusions were robust to methodological decisions made by the review authors:

- Risk of bias (restricting analysis to blinded studies at low risk of selection bias).

\section{RESULTS}

We identified 2133 records. After selection process, 37 studies met our inclusion criteria (figure 1). ${ }^{18-54}$

The characteristics of the included RCTs are presented in online supplementary material 1 . The 37 trials included 6348 women, with a sample size ranging from $25^{18}$ to $1156 .{ }^{19}$ 


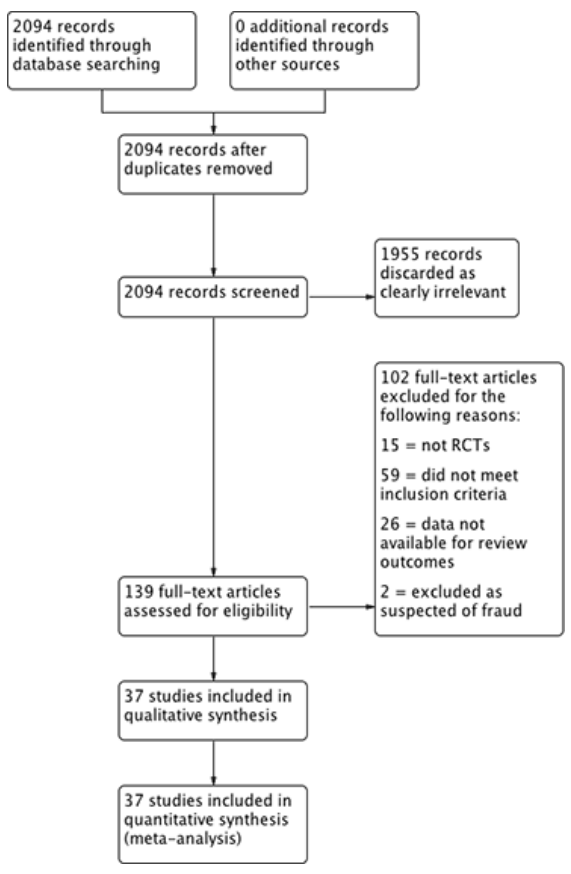

Figure 1 Study flow diagram. RCTs, randomised controlled trials.

Retrieved studies came from a wide range of inpatient and outpatient settings from different continents (North America, South America, Europe, Asia, Oceania and Africa). The trials recruited women aged 14 years and over with a diagnosis of PID according to the CDC criteria (pelvic or lower abdominal pain and one or more of the following clinical criteria: cervical motion tenderness, uterine tenderness or adnexal tenderness). ${ }^{2}$ Studies varied in the degree of disease severity of participants.

\section{Clinical cure in mild-moderate PID}

Two trials compared azithromycin versus doxycycline in mild-moderate PID. ${ }^{20} 21$ There was no clear evidence of a difference between azithromycin and doxycycline regimens (RR 1.18, $95 \%$ CI 0.89 to $1.55 ; 243$ women, 2 studies; $I^{2}=72 \%$; very low-quality evidence). Sensitivity analysis limited to the study at low risk of bias indicated that azithromycin was superior to doxycycline in achieving cure in mild-moderate PID (RR 1.35, $95 \%$ CI 1.10 to $1.67 ; 133$ women, 1 study; moderate-quality evidence). $^{21}$
Three studies compared quinolones versus cephalosporins. ${ }^{22-24}$ There was no clear evidence of a difference between the groups (RR $1.04,95 \%$ CI 0.98 to $1.10 ; 459$ women, 3 studies; $I^{2}=5 \%$; low-quality evidence).

Clinical cure in mild-moderate PID was evaluated in five studies comparing nitroimidazoles versus no nitroimidazoles. ${ }^{19}$ 25-28 In all the studies the nitroimidazole used was metronidazole. There was no conclusive evidence of a difference in effectiveness between metronidazole versus no use of metronidazole in mild-moderate PID (moderate-quality evidence; figure 2). Sensitivity analysis restricted to the two studies at low risk of bias did not substantially change the main findings (RR 1.06, 95\% CI 0.98 to 1.15 ; 1201 women, 2 studies; $\mathrm{I}^{2}=32 \%$; high-quality evidence). ${ }^{2526}$

Clindamycin plus aminoglycoside versus quinolone in mild-moderate PID was evaluated in one study, ${ }^{18}$ which showed no difference in effectiveness between the regimens (RR $0.88,95 \%$ CI 0.69 to $1.13 ; 25$ women, 1 study; $\mathrm{I}^{2}=0 \%$; very low-quality evidence).

Two studies compared clindamycin plus aminoglycoside versus cephalosporin in mild-moderate PID. ${ }^{29} 30$ There was no clear evidence of a difference between these regimens in the rates of cure for mild-moderate PID (RR 1.02, 95\% CI 0.95 to 1.09 ; 150 women, 2 studies; $\mathrm{I}^{2}=0 \%$; low-quality evidence).

\section{Clinical cure in severe PID}

One trial compared azithromycin versus doxycycline in severe PID. ${ }^{31}$ There was no clear evidence of a difference in the rates of cure between regimens using azithromycin or doxycycline to treat severe PID (RR 1.00, 95\% CI 0.96 to $1.05 ; 309$ women, 1 study; low-quality evidence).

Two studies compared quinolones versus cephalosporins, ${ }^{32} 33$ with no clear evidence of a difference in the rates of cure between regimens (RR $1.06,95 \%$ CI 0.91 to $1.23 ; 313$ women, 2 studies; $\mathrm{I}^{2}=7 \%$; low-quality evidence).

Eleven studies evaluated nitroimidazole in severe PID, and all studies used metronidazole. ${ }^{33-43}$ The difference in clinical cure rates between women treated with metronidazole and women not treated with it was small and was compatible with no effect (RR 0.96 , 95\% CI 0.92 to $1.01 ; 1383$ women, 11 trials; $\mathrm{I}^{2}=3 \%$; moderate-quality evidence; figure 3 ).

Two studies compared clindamycin plus aminoglycoside versus quinolone in severe PID. ${ }^{44}$ There was no clear evidence of a difference between these regimens in the rates of cure for severe

Clinical cure in mild-moderate pelvic inflammatory disease in regime
risk of bias, (-) high risk of bias, (?) unclear risk of bias. $\mathrm{M}-\mathrm{H}$, Mantel-Haenszel. 


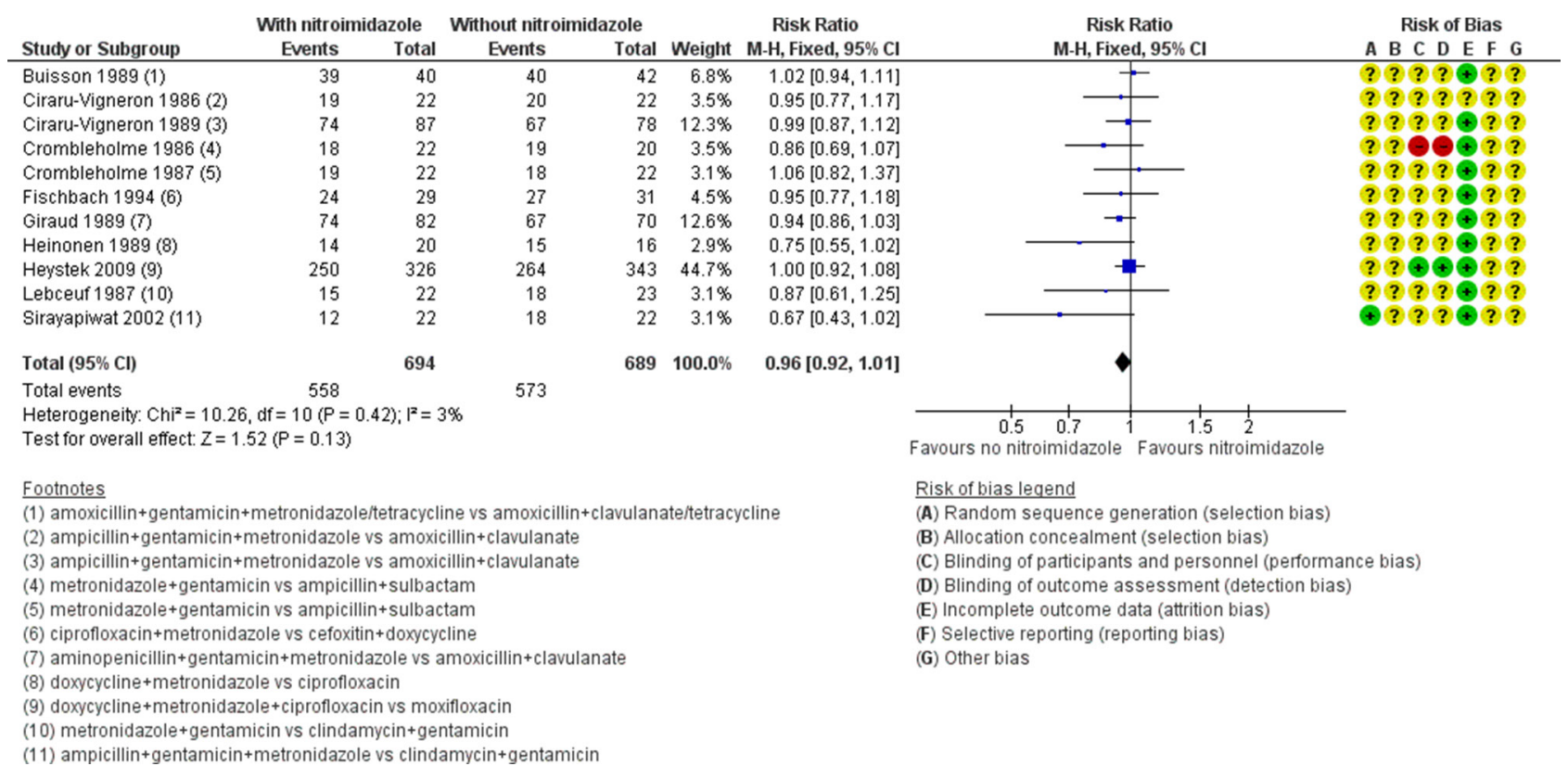

Figure 3 Clinical cure in severe pelvic inflammatory disease in regimens containing nitroimidazoles versus without nitroimidazoles. (+) low risk of bias, (-) high risk of bias, (?) unclear risk of bias. M-H, Mantel-Haenszel.

PID (RR 1.02, 95\% CI 0.87 to $1.19 ; 151$ women, 2 studies; $\mathrm{I}^{2}=0 \%$; low-quality evidence).

The use of clindamycin plus aminoglycoside versus cephalosporin in severe PID was evaluated in 10 studies. $^{29} 3046-53$ There was no clear evidence of a difference between these regimens in the rates of cure of severe PID (moderate-quality evidence; figure 4).

\section{Adverse events}

No clear evidence of a difference between regimens in antibiotic-related adverse events leading to discontinuation of therapy was observed for all comparisons.

\section{Quality of the evidence}

Most of the 37 included studies had unclear or high risk of bias in most domains, and only three were at low risk of bias in most domains. ${ }^{21} 2526$ The overall quality of the evidence ranged from very low to high, the main limitations being serious risk of bias (due to poor reporting of study methods and lack of blinding), serious inconsistency and serious imprecision. The only high-quality evidence was for the sensitivity analysis regarding the use (or not) of nitroimidazole. There was moderate-quality evidence in the sensitivity analysis regarding the use of azithromycin in mild-moderate cases of PID, in comparisons between the use or not of nitroimidazole

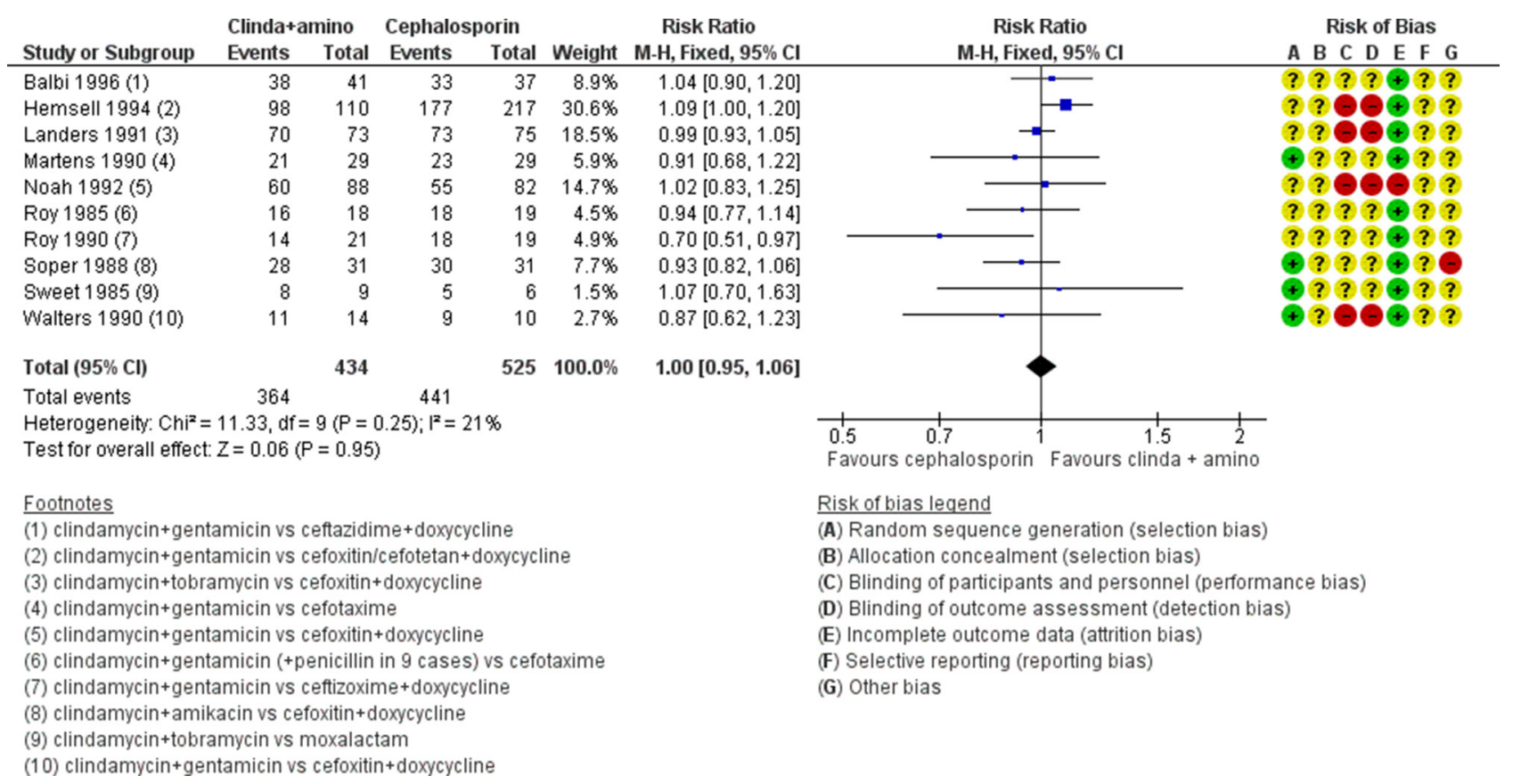

Figure 4 Clinical cure in severe pelvic inflammatory disease in regimens containing clindamycin plus aminoglycoside versus cephalosporin. (+) low risk of bias, (-) high risk of bias, (?) unclear risk of bias. M-H, Mantel-Haenszel. 
for curing mild-moderate or severe PID, and in comparisons between clindamycin plus aminoglycoside versus cephalosporins for curing severe PID.

\section{DISCUSSION}

Thirty-seven trials with 6348 women were included in the review. We found no clear evidence of a difference between any of the regimens studied in terms of effectiveness or safety. Within a sensitivity analysis of cases of mild-moderate PID for the comparison of macrolide (azithromycin) versus tetracycline (doxycycline), we identified a single study at low risk of bias which provided moderate-quality evidence that azithromycin was superior to doxycycline in achieving clinical cure. Some guidelines have recommended the use of nitroimidazoles for PID, ${ }^{211}$ but we found no conclusive evidence of a difference in outcomes between the use or not of nitroimidazoles (metronidazole) in successfully treating PID. There was also no clear evidence of a difference in rates of adverse effects between the regimens.

The applicability of the evidence to the target population (women of reproductive age diagnosed with PID) was broad because the included trials were conducted in different clinical settings and implemented varying diagnostic approaches. Additionally, the interventions analysed in the review are currently available and represent the most frequently used treatment regimens in current clinical practice. Given these factors, we consider that the evidence identified applies to a wide range of women with PID varying in disease severity, age, geographical location and diagnostic criteria, which provides external validity.

The trials included in this review cover a period of approximately 30 years and several countries, with 7 out of 37 studies being conducted after the year 2000. The searches for this review were last updated in July 2016 and new evidence may now be available. Considering the availability of additional studies, an update of this review is expected to take place in 2020. Little data are available on temporal variation in bacterial causes of PID because few countries systematically collect this information. Wide variations in the bacterial aetiology of PID may occur in different geographical areas and these may affect the choice of treatment, but few trials were carried out in low-income/middle-income countries. Although we consider that the results of this review are generalisable to a wide range of geographical locations, our conclusions may not be generalisable to low-income/middle-income countries.

An important limitation of this systematic review was the potential for measurement bias introduced by using the investigators' definitions of cure. This approach was necessary because of the wide variation in methods used and lack of a widely accepted objective outcome measure. The short-term follow-up in most studies prevented the identification of longterm sequelae. In addition, the inaccuracy of clinical diagnosis for PID and the wide variety of assessment criteria used for clinical cure may have reduced the power of the analysis to detect a clinically relevant effect. Some studies identified PID and endometritis separately, but these were pooled for our analysis. Data were lacking for several of our secondary outcomes. None of the included studies reported data on fertility or laparoscopic evidence of PID resolution, and data were very scant on length of hospital stay.

Microbiological clearance of C. trachomatis and N. gonorrhoeae and clinical resolution of symptoms are the usual outcomes used in clinical practice and the current review reflects this. ${ }^{12}$ The utility of other biological markers (eg, mediators of inflammation, 'new' bacteria) is being explored, but their use as outcome measures is not yet established even in a research setting. ${ }^{556}$ Bacterial sequencing and $16 \mathrm{~S}$ ribosomal RNA are also experimental, and their role in the diagnosis, or as prognostic markers, remains uncertain.

Increasing resistance in $N$. gonorrboeae has implications for the treatment of gonococcal PID and strongly suggests that only parenteral third-generation cephalosporins should be used in this situation, but this accounts for a very small proportion of PID overall. Studies have demonstrated that women with PID are often coinfected with other micro-organisms such as Mycoplasma genitalium, Trichomonas vaginalis, C. trachomatis and anaerobes. There is increasing recognition that $M$. genitalium is an important cause of PID, although only in a minority of women. However, few published studies (and no RCTs) have addressed this. ${ }^{1157}$ The utility of azithromycin as empirical treatment for M. genitalium is limited by increasing rates of resistance, and treatment with moxifloxacin is currently recommended for this group of patients. ${ }^{58} 59$ T. vaginalis has been associated with endometritis, but its role in PID remains uncertain. ${ }^{60}$ Bacterial vaginosis is not commonly associated with PID in prospective studies, although subgroups of women with bacterial vaginosis may be at higher risk, especially if coinfected with $N$. gonorrhoeae or C. trachomatis. ${ }^{61}$

Anaerobic bacteria are commonly identified in the fallopian tubes of women with PID, ${ }^{62}$ and most treatment guidelines include metronidazole to provide adequate microbiological cover. On meta-analysis we found that the addition of a nitroimidazole (metronidazole) did not improve short-term clinical outcomes of either mild-to-moderate or severe PID, which suggests that the other components of treatment regimens may be adequate. This is potentially important since nitroimidazoles commonly cause gastrointestinal side effects and may limit adherence to therapy. Nevertheless, some studies used antimicrobials with anaerobic cover, such as amoxicillin + clavulanate. ${ }^{28}$ The only study, in this systematic review, that did not use any antibiotic with anaerobic coverage found that women who did not receive nitroimidazole were more likely to experience clinical cure than those who received nitroimidazole. ${ }^{28}$ Of note, the study conducted by Burchell $e a l^{28}$ had a small sample size and lacked information to assess risk of bias. We were not able to assess longterm outcomes such as infertility or chronic pelvic pain, and it remains uncertain whether the use of nitroimidazoles affects the risk of these sequelae.

One previous meta-analysis, published in 1993, formed the basis for the CDC guidelines. ${ }^{63}$ The authors reported pooled clinical cure rates ranging from $75 \%$ to $94 \%$, which is similar to our updated review with overall rate of cure of $81 \%$. The uncertainty in using nitroimidazoles when treating PID is reflected in the current guidelines. The 2015 US CDC PID guideline ${ }^{2}$ advises that metronidazole be considered to provide additional anaerobic cover but does not mandate its use. The 2017 European IUSTI PID guideline ${ }^{11}$ and the 2018 BASHH PID guideline ${ }^{64}$ recommend the use of metronidazole but advise that it can be discontinued in those with mild to moderate symptoms if they develop drug-related side effects. Our analysis does not support the routine use of metronidazole in the treatment of women with mild to moderate PID and can be used to inform future guideline revisions. 


\section{Differences between the Cochrane review and the current review}

The current review is an abridged version of a Cochrane systematic review and presents the main findings and an enhanced discussion section. ${ }^{12}$ The secondary outcomes presented in the Cochrane review were microbiological clearance of C. trachomatis, microbiological clearance of N. gonorrhoeae, laparoscopic evidence of resolution of PID based on physician opinion, length of stay (for inpatient care) and rate of fertility. The results were similar for studies that reported microbiological clearance of C. trachomatis or N. gonorrhoeae, with clearance occurring in over $90 \%$ of women irrespective of regimen used. Length of stay varied across the studies, ranging from 3 days to 18 days. No data were found for laparoscopic evidence of resolution of PID and for rate of fertility.

\section{CONCLUSION}

We found no evidence that one regimen is more effective or safer than any other for the treatment of PID, and there is no clear evidence that the addition of nitroimidazoles is beneficial. Adherence to clinical treatment for PID is an important issue that should be considered when choosing a treatment regimen. Moderate-quality evidence from a single study at low risk of bias suggests that a macrolide (azithromycin) may be more effective than a tetracycline (doxycycline) for curing mild-moderate PID. There remains a need for high-quality RCTs to assess treatments for women with PID, particularly comparing regimens with or without the addition of nitroimidazoles and comparing azithromycin (eg, $1 \mathrm{~g}$ once a week for 2 weeks) with doxycycline. The lack of a consistent outcome measure to assess response to therapy is a major limitation, and there is a clear need for core outcome measures to be developed.

\section{Key messages}

- There is no clear evidence that any one of the currently recommended treatment regimens for pelvic inflammatory disease (PID) is superior to another.

- There is no evidence of improved efficacy when metronidazole is included within a treatment regimen, although this is currently recommended in some guidelines.

- There is a need for better understanding of the role of azithromycin in the treatment of PID, given uncertainty about its efficacy compared with doxycycline and concerns about inducing antimicrobial resistance.

\section{Handling editor Nicola Low}

Contributors RFS: coordination, study design, statistical analysis and review, writing the manuscript, grading the evidence in GRADE, and final approval of the manuscript. DGF: data collection, extraction, grading the risk of bias and final approval of the manuscript. RVD: data collection, extraction, grading the risk of bias, writing the manuscript and final approval of the manuscript. SF: data collection, extraction, grading the risk of bias, grading the evidence in GRADE and final approval of the manuscript. JR: study design, writing the manuscript, grading the evidence in GRADE and final approval of the manuscript.

Funding NIHR's 2012 Cochrane Review Incentive Scheme Award, UK.

Competing interests RFS, DGF, RVD and SF certify that they do not have any affiliations with, or involvement in, any organisation or entity with a direct financial interest in the subject matter of this review (eg, employment, consultancy, stock ownership, honoraria, expert testimony). JR declares the following interests: personal fees from GSK Pharma, Hologic Diagnostic and Janssen Pharma; ownership of shares in GSK Pharma and AstraZeneca Pharma; author of the UK and European Guidelines on Pelvic Inflammatory Disease; member of the European Sexually Transmitted Infections Guidelines Editorial Board; member of the National Institute for Health Research (NIHR) HTA Commissioning Board; NIHR Journals Editor; Editorial Board of the Cochrane Collaboration Sexually Transmitted Diseases Collaborative Review Group; and Sexually Transmitted Infections journal associate editor. The authors disclose that two of the authors (RFS and JR) had two publications used in the analysis. RFS and JR did not participate in the process for considering these studies for inclusion, data extraction, quality assessment and grading for risk of bias. The current article is based on a published Cochrane review.

Provenance and peer review Not commissioned; externally peer reviewed.

Open access This is an open access article distributed in accordance with the Creative Commons Attribution 4.0 Unported (CC BY 4.0) license, which permits others to copy, redistribute, remix, transform and build upon this work for any purpose, provided the original work is properly cited, a link to the licence is given, and indication of whether changes were made. See: http://creativecommons.org/ licenses/by/4.0

\section{REFERENCES}

1. Soper DE, disease Pinflammatory. Pelvic inflammatory disease. Obstet Gynecol 2010;116(2 Pt 1):419-28

2. Workowski KA, Bolan GA, Centers for Disease Control and Prevention. Sexually transmitted diseases treatment guidelines. MMWR Recomm Rep 2015;64(RR03):1-137.

3. Simms I, Rogers $P$, Charlett $A$. The rate of diagnosis and demography of pelvic inflammatory disease in general practice: England and Wales. Int J STD AIDS 1999:10:448-51.

4. Ross JD. Pelvic inflammatory disease. BMJ Clin Evid 2013;12:1606.

5. Blanchard JF, Moses S, Greenaway C, et al. The evolving epidemiology of chlamydial and gonococcal infections in response to control programs in Winnipeg, Canada. Am J Public Health 1998;88:1496-502.

6. Ness RB, Soper DE, Holley RL, et al. Effectiveness of inpatient and outpatient treatment strategies for women with pelvic inflammatory disease: results from the Pelvic Inflammatory Disease Evaluation and Clinical Health (PEACH) randomized trial. Am J Obstet Gynecol 2002;186:929-37.

7. Ness RB, Trautmann G, Richter HE, et al. Effectiveness of treatment strategies of some women with pelvic inflammatory disease: a randomized trial. Obstet Gynecol 2005;106:573-80.

8. Mahafzah AM, Al-Ramahi MQ, Asa'd AM, et al. Prevalence of sexually transmitted infections among sexually active Jordanian females. Sex Transm Dis 2008:35:607-10.

9. Trent M, Ellen JM, Frick KD. Estimating the direct costs of pelvic inflammatory disease in adolescents: a within-system analysis. Sex Transm Dis 2011;38:326-8.

10. Aghaizu A, Adams EJ, Turner $K$, et al. What is the cost of pelvic inflammatory disease and how much could be prevented by screening for chlamydia trachomatis? cost analysis of the Prevention of Pelvic Infection (POPI) trial. Sex Transm Infect 2011;87:312-7.

11. Ross J, Guaschino S, Cusini M, et al. 2017 European guideline for the management of pelvic inflammatory disease. Int J STD AIDS 2018;29:108-14.

12. Savaris RF, Fuhrich DG, Duarte RV, et al. Antibiotic therapy for pelvic inflammatory disease. Cochrane Database Syst Rev 2017;4:CD010285.

13. Higgins JPT, Green S, 2011. Cochrane handbook for systematic reviews of interventions version 5.1.0. Available from: handbook.cochrane.org

14. Savaris RF, Ross J, Fuhrich DG, et al. Antibiotic therapy for pelvic inflammatory disease(PID) [Protocol]. Cochrane Database Syst Rev 2013;1:CD010285.

15. Andrews JC, Schünemann HJ, Oxman AD, et al. GRADE guidelines: 15. Going from evidence to recommendation-determinants of a recommendation's direction and strength. J Clin Epidemiol 2013:66:726-35.

16. Nordic Cochrane Centre. Review Manager 5 (RevMan 5). Version 5.3. Copenhagen: Nordic Cochrane Centre, The Cochrane Collaboration, 2014.

17. Altman DG. Confidence intervals for the number needed to treat. $B M J$ 1998;317:1309-12.

18. Apuzzio JJ, Stankiewicz R, Ganesh V, et al. Comparison of parenteral ciprofloxacin with clindamycin-gentamicin in the treatment of pelvic infection. Am J Med 1989;87(5A):S148-51.

19. Aşicioğlu O, Gungorduk K, Ozdemir A, et al. Single daily dose of moxifloxacin versus ofloxacin plus metronidazole as a new treatment approach to uncomplicated pelvic inflammatory disease: a multicentre prospective randomized trial. Eur J Obstet Gynecol Reprod Biol 2013;171:116-21.

20. Malhotra M, Sharma JB, Batra S, et al. Ciprofloxacin-tinidazole combination, fluconazole- azithromicin-secnidazole-kit and doxycycline- metronidazole combination therapy in syndromic management of pelvic inflammatory disease: a prospective randomized controlled trial. Indian J Med Sci 2003;57:549-55.

21. Savaris RF, Teixeira LM, Torres TG, et al. Comparing ceftriaxone plus azithromycin or doxycycline for pelvic inflammatory disease: a randomized controlled trial. Obstet Gynecol 2007;110:53-60. 
22. Wendel GD, Cox SM, Bawdon RE, et al. A randomized trial of ofloxacin versus cefoxitin and doxycycline in the outpatient treatment of acute salpingitis. Am J Obstet Gynecol 1991;164(5 Pt 2):1390-6.

23. Martens MG, Gordon S, Yarborough DR, et al. Multicenter randomized trial of ofloxacin versus cefoxitin and doxycycline in outpatient treatment of pelvic inflammatory disease. Ambulatory PID Research Group. South Med J 1993;86:604-10.

24. Arredondo JL, Diaz V, Gaitan H, et al. Oral clindamycin and ciprofloxacin versus intramuscular ceftriaxone and oral doxycycline in the treatment of mild-to-moderate pelvic inflammatory disease in outpatients. Clin Infect Dis 1997;24:170-8.

25. Judlin $P$, Liao Q, Liu Z, et al. Efficacy and safety of moxifloxacin in uncomplicated pelvic inflammatory disease: the MONALISA study. BJOG 2010;117:1475-84.

26. Ross JD, Cronjé HS, Paszkowski T, et al. Moxifloxacin versus ofloxacin plus metronidazole in uncomplicated pelvic inflammatory disease: results of a multicentre, double blind, randomised trial. Sex Transm Infect 2006;82:446-51.

27. Tison E, Marpeau L, Pigné A. Treatment of acute non-chlamydial salpingitis. Study of the efficacy and tolerance of a single-therapy antibiotic: Augmentin. J Gynecol Obstet Biol Reprod 1988;17:513-9.

28. Burchell HJ, Cronjé HS, de Wet Jl. Efficacy of different antibiotics in the treatment of pelvic inflammatory disease. S Afr Med J 1987;72:248-9.

29. Sweet RL, Ohm-Smith M, Landers DV, et al. Moxalactam versus clindamycin plus tobramycin in the treatment of obstetric and gynecologic infections. Am J Obstet Gynecol 1985;152(7 Pt 1):808-17.

30. Walters MD, Gibbs RS. A randomized comparison of gentamicin-clindamycin and cefoxitin-doxycycline in the treatment of acute pelvic inflammatory disease. Obstet Gynecol 1990;75:867-72.

31. Bevan CD, Ridgway GL, Rothermel CD. Efficacy and safety of azithromycin as monotherapy or combined with metronidazole compared with two standard multidrug regimens for the treatment of acute pelvic inflammatory disease. J Int Med Res 2003;31:45-54

32. Okada H, Yamamoto T, Yasuda J, et al. Comparative clinical study on ciprofloxacin and cefroxadine in the treatment of infections in obstetrics and gynecology. Chemotherapy 1988;36:821-57.

33. Fischbach F, Deckardt R, Graeff H. Ciprofloxacin/Metronidazol vs. Cefoxitin/Doxycyclin: Vergleich zweier Therapieschemata zur Behandlung der akuten pelvinen Infektion. Geburtshilfe Frauenheilkd 1994;54:337-40.

34. Ciraru-Vigneron N, Bercau G, Sauvanet E, et al. The drug combination amoxicillinclavulanic acid compared to the triple combination ampicillin-gentamicinmetronidazole in the treatment of severe adnexal infections. Pathol Biol 1986;34(5):665-8

35. Cirau-Vigneron N, Barrier J, Becue J, et al. Amoxycillin/clavulanic acid ('Augmentin') compared with a combination of aminopenicillin, aminoglycoside and metronidazole in the treatment of pelvic inflammatory disease. Pharmatherapeutica 1989:5:312-9.

36. Crombleholme W, Landers D, Ohm-Smith M, et al. Sulbactam/ampicillin versus metronidazole/gentamicin in the treatment of severe pelvic infections. Drugs 1986;31(Suppl 2):11-13

37. Crombleholme WR, Ohm-Smith M, Robbie MO, et al. Ampicillin/sulbactam versus metronidazole-gentamicin in the treatment of soft tissue pelvic infections. Am J Obstet Gynecol 1987;156:507-12.

38. Buisson P, Mulard C, Baudet J, et al. Treatment of upper genital infections in women. Multicenter study of the comparative efficacy and tolerance of an amoxicillinclavulanic acid combination and of a triple antibiotic combination. Rev Fr Gynecol Obstet 1989;84:699-703.

39. Giraud JR, Chartier M, Ciraru Vigneron N, et al. A comparison of the efficacy of and tolerance to Augmentin used alone and as one of three drugs used to treat acute upper genital tract infections. Results of a multicentre trial 152 cases. Contraception Fertilite Sexualite 1989;17:941-8.

40. Heinonen PK, Teisala K, Miettinen A, et al. A comparison of ciprofloxacin with doxycycline plus metronidazole in the treatment of acute pelvic inflammatory disease. Scand I Infect Dis Supp/ 1989;60:66-73.

41. Heystek M, Ross JD, PID Study Group. A randomized double-blind comparison of moxifloxacin and doxycycline/metronidazole/ciprofloxacin in the treatment of acute, uncomplicated pelvic inflammatory disease. Int J STD AIDS 2009;20:690-5.
42. Lebceuf D, Rousset D, Cacault JA, et al. Prospective randomized study comparing the efficacy and tolerance of clindamycin-gentamycin versus metronidazole-gentamycin in acute utero-adnexal infections in hospitalized patients. Rev Fr Gynecol Obstet 1987:82:9-15

43. Sirayapiwat $\mathrm{P}, \mathrm{Chaithongwongwatthana} \mathrm{S}$, Tirawatnapong $\mathrm{S}$. Triple therapies versus clindamycin plus gentamicin in the treatment of acute pelvic inflammatory disease, a randomized controlled trial. Thai J Obstet Gynaecol 2002;14:215-21.

44. Crombleholme WR, Schachter J, Ohm-Smith M, et al. Efficacy of single-agent therapy for the treatment of acute pelvic inflammatory disease with ciprofloxacin. Am J Med 1989;87(5A):S142-7.

45. Thadepalli H, Mathai D, Scotti R, et al. Ciprofloxacin monotherapy for acute pelvic infections: a comparison with clindamycin plus gentamicin. Obstet Gynecol 1991;78:696-701.

46. Roy S, Wilkins J. Cefotaxime in the treatment of female pelvic soft tissue infections. Infection 1985;13(S1):S56-S61.

47. Roy S, Wilkins J, March CM, et al. A comparison of the efficacy and safety of ceftizoxime with doxycycline versus conventional CDC therapies in the treatment of upper genital tract infection with or without a mass. Clin Ther 1990;12(Suppl C):53-73.

48. Soper DE, Despres B. A comparison of two antibiotic regimens for treatment of pelvic inflammatory disease. Obstet Gynecol 1988;72:7-12.

49. Martens MG, Faro S, Hammill H, et al. Comparison of cefotaxime, cefoxitin and clindamycin plus gentamicin in the treatment of uncomplicated and complicated pelvic inflammatory disease. J Antimicrob Chemother 1990;26(suppl A):37-43.

50. Noah ML. Comparative evaluation of clindamycin/gentamicin and cefoxitin/ doxycycline for treatment of pelvic inflammatory disease: a multi-center trial. The European Study Group. Acta Obstet Gynecol Scand 1992;71:129-34.

51. Landers DV, Wolner-Hanssen P, Paavonen J, et al. Combination antimicrobial therapy in the treatment of acute pelvic inflammatory disease. Am J Obstet Gynecol 1991;164:849-58.

52. Hemsell DL, Little BB, Faro S, et al. Comparison of three regimens recommended by the Centers for Disease Control and Prevention for the treatment of women hospitalized with acute pelvic inflammatory disease. Clin Infect Dis 1994;19:720-7.

53. Balbi G, Piscitelli V, Di Grazia F, et al. Acute pelvic inflammatory disease: comparison of therapeutic protocols. Minerva Ginecol 1996;48(1-2):19-23.

54. Hoyme UB, Ansorg R, Von Recklinghausen G. Quinolones in thetreatment of uncomplicated salpingitis: ofloxacin/metronidazole vs. gentamicin/clindamicin. Arch Gynecol Obstet 1993;254:607-8

55. Haggerty $C L$, Totten PA, Tang G, et al. Identification of novel microbes associated with pelvic inflammatory disease and infertility. Sex Transm Infect 2016;92:441-6.

56. Taylor BD, Zheng X, Darville T, et al. Whole-Exome Sequencing to Identify Novel Biological Pathways Associated With Infertility After Pelvic Inflammatory Disease. Sex Transm Dis 2017;44:36-42.

57. Manhart LE. Mycoplasma genitalium: An emergent sexually transmitted disease? Infect Dis Clin North Am 2013;27:779-92.

58. Bissessor M, Tabrizi SN, Twin J, et al. Macrolide resistance and azithromycin failure in a Mycoplasma genitalium-infected cohort and response of azithromycin failures to alternative antibiotic regimens. Clin Infect Dis 2015;60:1228-36.

59. Read TR, Fairley CK, Tabrizi SN, et al. Azithromycin 1.5g Over 5 Days Compared to $1 \mathrm{~g}$ Single Dose in Urethral Mycoplasma genitalium: Impact on Treatment Outcome and Resistance. Clin Infect Dis 2017;64:250-6.

60. Cherpes TL, Wiesenfeld HC, Melan MA, et al. The associations between pelvic inflammatory disease, Trichomonas vaginalis infection, and positive herpes simplex virus type 2 serology. Sex Transm Dis 2006;33:747-52.

61. Ness RB, Hillier SL, Kip KE, et al. Bacterial vaginosis and risk of pelvic inflammatory disease. Obstet Gynecol 2004;104:761-9.

62. Hebb JK, Cohen CR, Astete SG, et al. Detection of novel organisms associated with salpingitis, by use of $16 \mathrm{~S}$ rDNA polymerase chain reaction. J Infect Dis 2004:190:2109-20.

63. Walker CK, Kahn JG, Washington AE, et al. Pelvic inflammatory disease: meta analysis of antimicrobial regimen efficacy. J Infect Dis 1993;168:969-78.

64. Ross J, Cole M, Evans C, 2018. United Kingdom national guideline for the management of pelvic inflammatory disease. https://www.bashhguidelines.org/media/ 1170/pid-2018.pdf (accessed 28 Aug 2018). 